\title{
Treatment of Isolated Sternal Fracture with Ultrasound-Guided Paravertebral Nerve Block: a Case Report and Literature Review
}

Jun-Mo Park ${ }^{1}$, Hyunjeong Kim ${ }^{1}$

Department of Anesthesiology and Pain Medicine, School of Medicine, Kyungpook National University, Kyungpook National University Hospital, Daegu, Korea

In the case of isolated sternal fractures, conservative treatment with analgesics is common, but pain can persist for more than 10 weeks, which can significantly interfere with daily life. Ultrasound-guided paravertebral nerve block is reported to be a successful means of pain control in patients with chest wall injury or rib fracture.

A 70-year-old female patient presented with anterior chest pain that had persisted for 2 weeks despite conservative treatment. Sagittal reconstruction chest computed tomography and sternum lateral oblique x-ray revealed an isolated sternal fracture. An ultrasound-guided bilateral paravertebral nerve block was performed for pain control. After performing the procedure twice at a 1-week interval, the patient reported complete pain alleviation, and no other problems were observed over the 3-month follow-up period.

Ultrasound-guided bilateral paravertebral nerve block can help patients with isolated sternal fractures to manage pain and return to normal activities sooner than with oral analgesics.

Key Words: Fracture, Nerve block, Pain management, Sternum, Ultrasonography

Sternal fractures are not uncommon and have been reported to occur in $3.7 \%$ of patients hospitalized after a traffic accident. ${ }^{1}$ Before the use of seatbelts became mandatory, a sternal fracture was a marker for high-energy trauma. After the use of safety belts was legislated, the number of patients surviving traffic collisions increased, accompanied by an increase in patients presenting with sternal fractures as a result of these accidents.

The morbidity and mortality rates of sternal fractures are determined by associated injuries to the internal thoracic organs, with isolated sternal fractures considered benign lesions in the absence of accompanying cardio-respiratory damage. ${ }^{1,2}$ More than $95 \%$ of sternal fractures are treated conservatively. ${ }^{2}$ The most important factor in the treatment of isolated sternal fractures is adequate pain control, usually managed with oral analgesics. ${ }^{3}$

Few studies, however, have been published regarding the functional effects of isolated sternal fractures on daily activities. According to one study, although chest pain was the most fre-

Corresponding Author: Jun-Mo Park, Department of Anesthesiology and Pain Medicine, Kyungpook National University Chilgok Hospital, 807 Hoguk-ro, Buk-gu, Daegu 41404, Korea

\section{(6) (1) (3)}

Articles published in Kosin Medical Journal are open-access, distributed under the terms of the Creative Commons Attribution Non-Commercial License (http://creativecommons.org/licenses/by-nc/4.0/) which permits unrestricted non-commercial use, distribution, and reproduction in any medium, provided the original work is properly cited. 
quently reported and persistent symptom, continuing for an average of 10.9 weeks, the duration of pain was significantly prolonged in patients older than 50 years of age presenting with an isolated sternal fracture. ${ }^{4}$ This prolonged chest pain often necessitates the administration of oral analgesics for a relatively long period, but this long-term use can be limited by the side effects of non-steroidal anti-inflammatory drugs (NSAIDs) and opioids. ${ }^{5}$ In particular, older patients may have underlying medical conditions that contraindicate analgesic use. Other patients may have to return to their daily lives before the pain has subsided, which can be difficult to do when undergoing conservative treatment alone.

We report a case of successful management of pain in a patient with an isolated sternal fracture using an ultrasound-guided bilateral paravertebral nerve block.

\section{CASE}

A 70-year-old female patient presented with anterior chest pain that had persisted for 2 weeks. The patient had no pertinent medical history other than 10 years of anti-hypertensive drug medication. The patient reported a fall 2 weeks before visiting the pain clinic, hitting her chest against a stone, followed by chest pain. The pain was less severe when she remained still but worsened when she coughed or spread her arms, and she reported difficulty with bending her chest forward or turning over in bed. The patient's visual analog scale (VAS) score was 7 out of 10 .
She was examined at a local clinic near her home and was told that her sternum had cracked. That doctor recommended rest and prescribed an NSAID medication. However, her symptoms had not improved, and she complained that the NSAID was causing facial swelling and heartburn. In addition, the patient was planning an overseas trip with her family in 2 weeks. She visited our clinic to address her pain so she could travel unencumbered.

A sternal fracture was already suspected at the other facility, and the pain had persisted even after 2 weeks of adequate treatment. We thereby performed chest computed tomography (CT) including sagittal reconstruction, $x$-rays of the sternum lateral and lateral oblique aspects, electrocardiography (ECG), and a cardiac enzyme test to check for any accompanying injuries. A thorough physical examination was also conducted on the patient, and no specific abnormal signs were found other than intense, localized tenderness at the mid portion of the sternal body. A recent fracture was observed at the sternum between the third and fourth ribs on the sagittal reconstruction chest $\mathrm{CT}$ and sternum lateral oblique x-ray (Fig. 1A, 1B). There was mild soft tissue edema around the fractured area, but no hemorrhage, and no soft tissue injury that would indicate a pathologic fracture. No abnormalities were found on the ECG or cardiac enzyme test. As such, we decided to perform an ultrasoundguided bilateral paravertebral nerve block at the space between the third and fourth ribs to allow the patient to return to normal daily life sooner, particularly, to travel abroad with her family after 


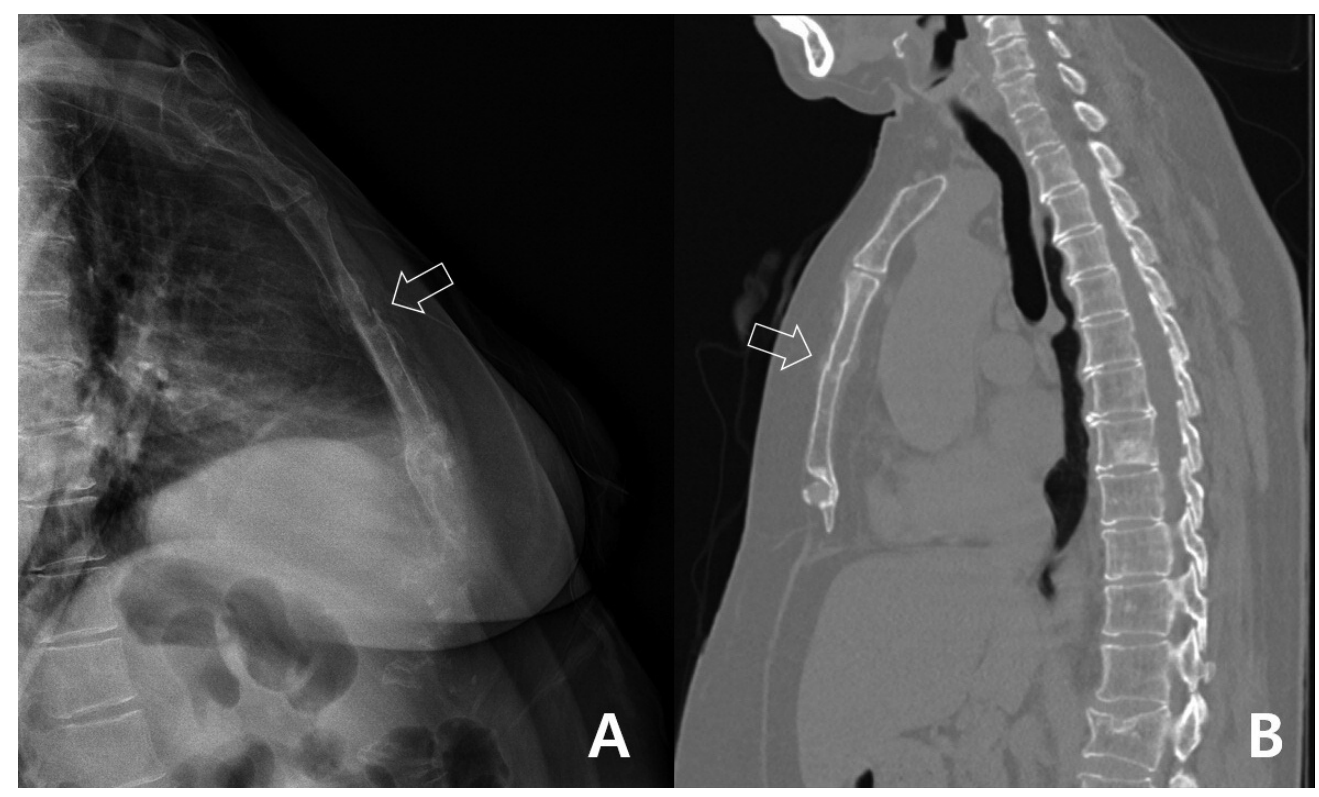

Fig. 1. (A) Sternum lateral oblique x-ray. (B) Sagittal reconstruction chest CT. Arrows indicate fracture site.

2 weeks. The patient was placed in the prone position, the third and fourth ribs were visualized with C-arm guidance (Fig. 2A), and the skin was marked along the running direction of each rib (Fig. 2B). After sterilization of the skin, a curved transducer (1.5-4.6 MHz) for a GE Logiq7 ultrasound machine (Tokyo, Japan) was placed parallel to the direction indicated on the skin and moved medially to check the spinous and transverse processes of the third thoracic vertebra. The transducer was then moved laterally along the skin markings to locate the thoracic paravertebral space for injection (Fig. 2C). The transducer was moved slightly in the cephalad and caudal directions perpendicular to the direction of the rib indicated on the skin to confirm that the rib was excluded from the path of the needle (Fig. 3A). A 10-cm, 22-gauge SonoPlex cannula with facet grinding (Pajunk $\mathrm{GmbH}$, Geisingen, Germany) was inserted in the lateral to medial direction using an in-plane technique. After it was confirmed that the needle was placed in the region under the transverse process and that no blood or air was withdrawn during negative aspiration, a mixture of $10 \mathrm{~mL}$ of $0.5 \%$ lidocaine and $20 \mathrm{mg}$ of methylprednisolone acetate was slowly injected. During the injection, concomitant anterior movement of the parietal pleura was checked by ultrasound to confirm that the drug was injected into the correct location (Fig. 3B). After completing this procedure on one side, it was repeated on the other side.

Thirty minutes after the procedure, the patient's VAS score of the pain caused by coughing or spreading her arms was reduced to 2 out of 10 , without any complications. Since the patient complained of side effects from the NSAID, tramadol and a skeletal muscle relaxant were prescribed, and the patient was asked to return for a follow-up in 1 week. After 1 week, the patient 


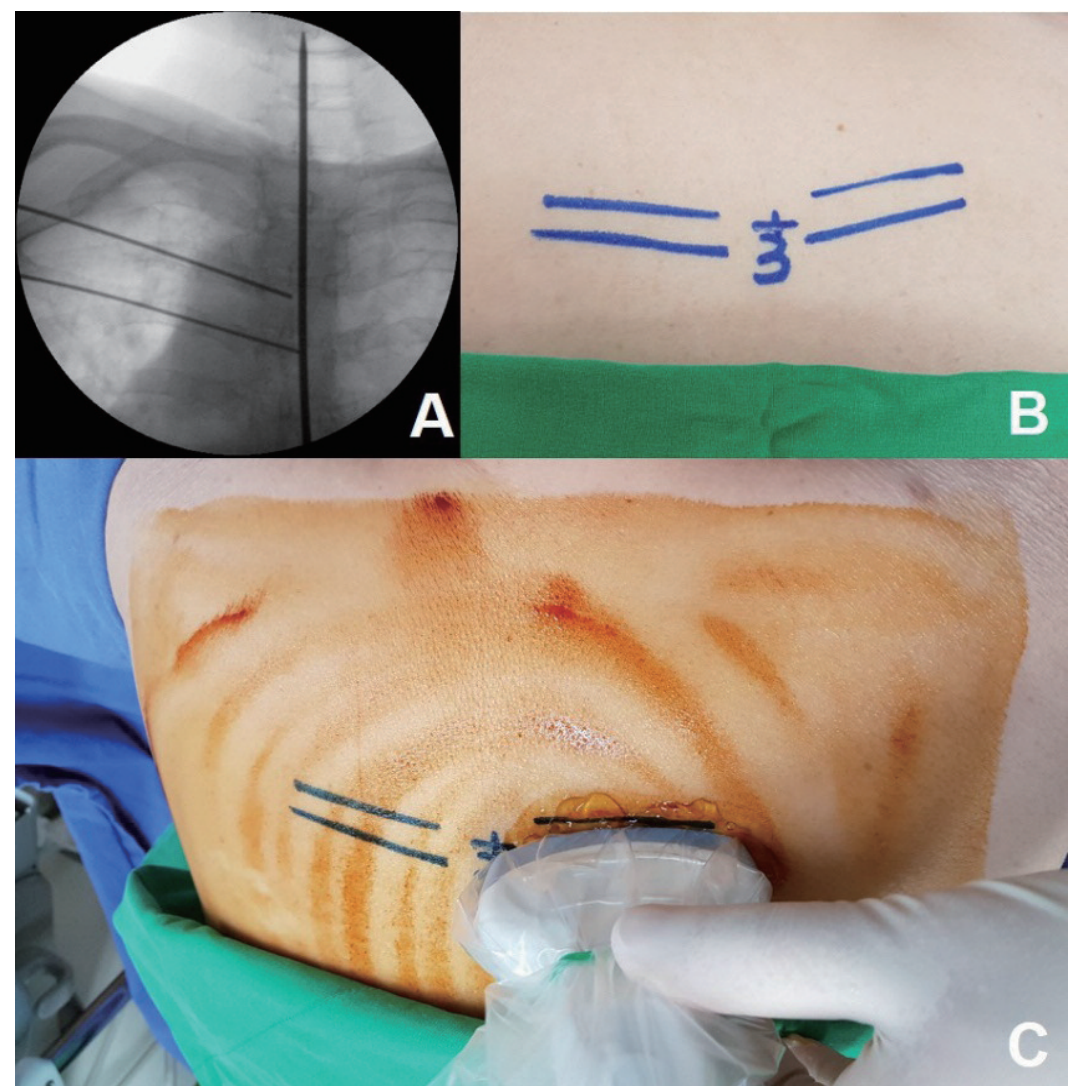

Fig. 2. (A) C-arm view showing the direction of the third and fourth ribs. (B) Skin was marked along the running direction of the third and fourth ribs. (C) Curved transducer was placed parallel to the direction indicated on the skin and moved medially and laterally along the skin markings and cephalad and caudally to locate the thoracic paravertebral space for injection.

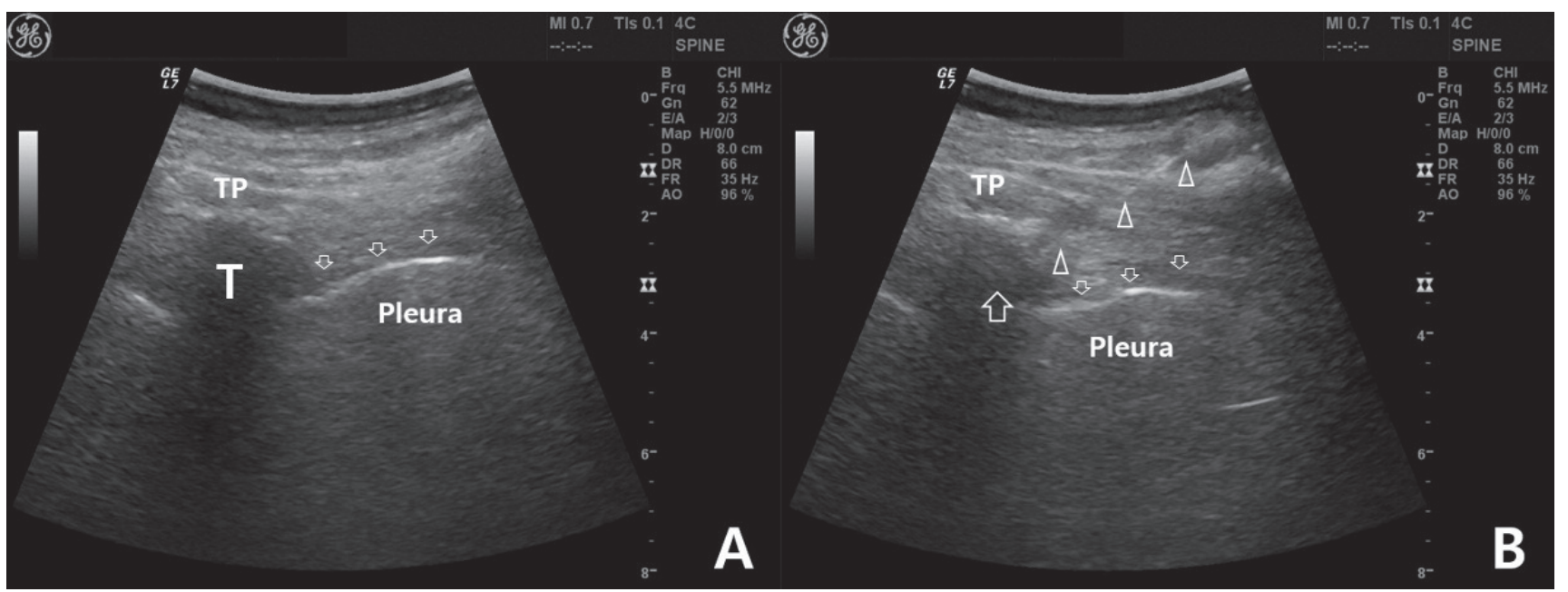

Fig. 3. (A) Ultrasound view showing the ribs were excluded from the path of the needle. (B) Ultrasound view showing anterior movement of the parietal pleura (small arrow). T: target region, TP: transverse process, needle (arrow head), tip of needle (large arrow). 
reported that her VAS score was about 1 or 2 out of 10 for most of the day. She also did not complain of any particular side effects of the prescribed drugs. Since the patient was concerned that the pain would recur during the planned overseas trip with her family 1 week later, the procedure was repeated, and the same drugs were prescribed. The patient followed up with us 2 weeks after the trip and reported that she had not felt any pain during the trip and that she had since stopped taking the pain medication because the pain had completely subsided. At her 3month follow-up, the patient reported a return to normal activities without pain, and no abnormalities were found on lateral and lateral oblique $\mathrm{x}$ rays of the sternum; thus, her treatment was terminated.

Approval of this study was waived from the Ethics Committee of Kyungpook National University Chilgok Hospital based upon their policy on case reports. The authors obtained written consent from the patient to publish this case report.

\section{DISCUSSION}

In the present case report, after ultrasoundguided bilateral paravertebral nerve block was performed twice, the patient's pain was successfully relieved and she was able to return to daily life early. This is the first report on pain management of an isolated sternal fracture with an ultrasound-guided bilateral paravertebral nerve block.

In 2014, through a retrospective analysis of a total of 32,746 cases of injuries including sternal fracture between 2007 and 2010 using the National Trauma Data Bank, Yeh et al. reported that $84 \%$ of sternal fractures were associated with car accidents, with sternal fracture confirmed in $3.7 \%$ of all patients hospitalized due to a collision. ${ }^{1}$ According to Oyetunji et al., the most common cause of sternal fracture is car accidents, and the second most common cause is falls. ${ }^{6}$ Other common causes are motorcycle injuries, pedestrian injuries from cars, and cyclist injuries. They also reported that most traumatic sternal fractures occur as one of multiple traumatic injuries rather than in isolation. Sternal fracture is often associated with severe blunt trauma and frequently associated with severe intrathoracic and vertebral injuries. The injuries most commonly associated with sternal fracture are rib fracture $(57.8 \%)$, lung contusion, closed pneumothorax or hemothorax, closed thoracic or lumbar vertebra fracture, concussion, and blunt cardiac injury. The morbidity and mortality rates associated with sternal fracture are determined by the level of internal thoracic organ damage accompanying the injury, with a mortality rate ranging from 4 to $45 \% .{ }^{2}$ For this reason, it is important to check for the presence of accompanying injuries in all patients presenting with sternal fracture, and current protocols call for cardiac monitoring, serial ECG, and hospitalization for further examination as needed. ${ }^{7}$ However, isolated sternal fractures without other thoracic injury do not necessarily require this level of care. In the absence of accompanying cardio-pulmonary complications, an isolated sternal frac- 
ture is considered a benign injury. ${ }^{2,3}$

Previously, before the use of routine CT for diagnosis and evaluation in blunt trauma patients, lateral chest $\mathrm{x}$-ray was considered the most useful method for diagnosing sternal fractures, and sternal fractures were considered clinically significant injuries associated with high morbidity and mortality rates. ${ }^{8}$ According to Perez et al., most sternal fractures can only be observed on $\mathrm{CT}$, are clinically insignificant, require no change in treatment protocol, and are accompanied by a low mortality incidence. ${ }^{8}$ Terry et al. noted that CT remains the best diagnostic imaging method for trauma patients for the rapid identification of multiple injuries. ${ }^{9}$ However, due to the curvature of the anterior chest wall, an axial image may miss a sternal injury, as the sternum may be excluded from the image. They suggested including a sagittal reconstruction image to increase agreement among imaging physicians and confidence in the diagnosis and recommended adding sagittal reconstruction to the standard chest trauma CT protocol. The sagittal reconstruction image can be appended to the exam with little or no additional cost to the patient or the institution and without exposing the patient to additional radiation. In the present case, the sagittal reconstruction image (Fig. 1B) was used to diagnose the isolated sternal fracture and check for the presence of accompanying injuries with a high level of accuracy.

Sternal fracture or dislocation usually occurs in the sagittal plane, rendering the lateral view of the sternum best suited to accurate diagnosis. ${ }^{8}$ Conventional radiography can sometimes fail to visualize such fractures. In addition, the lateral view of the sternum is an unattractive option for critically injured patients, especially those with multiple injuries. As such, ultrasound-based diagnosis is increasingly relied upon in emergency departments for sternal fracture patients and other types of trauma patients. ${ }^{10}$ The use of ultrasound to detect sternal fractures can be as sensitive or even twice as sensitive as conventional radiography. Ultrasonography allows physicians to quickly and easily identify the presence or absence of sternal fracture in trauma patients.

Sternal fracture can cause short- and long-term sequelae. De Oliveria et al. reported that persisting chest pain was the most common short-term sequelae and the duration of chest pain after sternal fracture was between 2 and 28 weeks, with an average of 8 to 12 weeks (mean period of 10.9 weeks) for all age groups, and noted that pain durations were significantly prolonged in patients over 50 years of age. ${ }^{4}$ Chest pain can worsen in cold weather or while performing certain maneuvers such as lifting, walking, or lying down. They also reported that the mean length of time that had to be taken off work was 5.1 weeks. Delayed complications from sternal fractures are uncommon; however, some do present with delayed complications and poor functional outcomes. Racine et al. reported that delayed hemothorax occurred in $12.5 \%$ of patients with sternal fracture. ${ }^{11}$ However, the clinical significance of delayed hemothorax remains uncertain. They reported that the proportion of patients with moderate to severe disability was significantly higher for sternal fracture than other types of 
minor thoracic trauma.

According to the systematic review of the literature published between 1990 and 2017 by Klei et al., the majority of patients with sternal fractures (more than 95\%) received conservative treatments such as bed rest, oral analgesics, and orthotics. ${ }^{2}$ They found that proper pain control was critical, because sternal fractures can cause pain during breathing that results in respiratory complications due to respiratory insufficiency. Although it is often necessary to take oral analgesics for an extended period of time in the conservative treatment of sternal fractures due to prolonged pain, long-term treatment may not be possible due to the side effects of NSAIDs or opioids. ${ }^{5}$ In particular, older patients or patients with underlying diseases may not be good candidates for the NSAIDs or opioids used in conservative treatment. ${ }^{7}$ Because of these issues, several new treatments have been introduced to treat these patients, including infiltration of local anesthetics directly into the fracture site with a subcutaneous cannula, periosteally positioned catheter, or sternal catheter. ${ }^{7}$ Recent developments of new procedures using ultrasound are also being applied to the treatment of patients with sternal fracture. ${ }^{12,13}$

Recently, ultrasound has been used not only for the diagnosis of sternal fracture but also for the treatment in more and more cases. ${ }^{12,13}$ In the present case, only 2 weeks after sternal fracture, conservative management with oral analgesics was needed. However, the pain was not controlled by NSAIDs, and NSAIDs could no longer be taken due to their side effects. Moreover, in order to be able to safely travel abroad, it was necessary to return to daily life early. The ultrasound-guided bilateral paravertebral nerve block was chosen after taking the patient's medical background and personal circumstances into account and performed to help the patient return to normal activities sooner.

The ultrasound-guided paravertebral nerve block is used during surgeries for breast cancer, inguinal hernia repair, lithotripsy, and video-assisted thoracic surgery; however, it is most commonly used for perioperative pain management and pain management in patients with multiple rib fractures. ${ }^{14}$ Thoracic paravertebral block is a method of injecting drugs into the thoracic vertebrae close to the spinal nerve from the intervertebral foramen. This procedure allows for the ipsilateral somatic and sympathetic nerve block, and characteristically, the injected drug spreads up and down the injection site to enable blockade in multiple dermatomes. The intercostal nerves innervate the costotransverse joints, the ribs (112), the costosternal joints, and the sternum. Since the intercostal nerve originates from the thoracic spinal nerve, it was able to block the corresponding intercostal nerve that innervated the fractured sternal region. Unlike the intercostal nerve block, one procedure on one side was sufficient because the drug spread to the level below or above the injection site. This procedure has the advantage of being easier and more familiar because it has been used by more and more anesthesiologists as well as pain medicine doctors in recent years.

In most cases, conservative treatment is all that 
is necessary, but some sternal fracture patients do require surgical treatment. Recently, surgical treatment has been recommended when the fracture is displaced, unstable, causing severe pain, or associated with flail chest threatening cardiopulmonary function. ${ }^{7}$ In chronic cases, surgical treatment is considered only when persistent pain, non-union, or cosmetically unacceptable external appearance is associated with the case. Most researchers do not support surgical treatment without a definite indication.

In conclusion, isolated sternal fracture is considered a benign injury in the absence of other associated cardio-pulmonary conditions. Conservative treatment is typical, and delayed complications rarely occur. However, conservative treatment may be insufficient in older patients, those with conditions contraindicating the use of oral analgesics, or those that need to quickly resume normal activities. In these cases, an ultrasound-guided bilateral paravertebral nerve block may help control pain and allow the patient to return to normal activities sooner than with treatment with oral analgesics alone.

\section{REFERENCES}

1. Yeh DD, Hwabejire JO, DeMoya MA, Alam HB, King DR, Velmahos GC. Sternal fracture--an analysis of the National Trauma Data Bank. J Surg Res 2014;186:39-43.

2. Klei DS, de Jong MB, Öner FC, Leenen LPH, van Wessem KJP. Current treatment and outcomes of traumatic sternal fractures-a systematic review. Int Orthop 2019;43:1455-64.

3. Sadaba JR, Oswal D, MunschCM. Management of isolated sternal fractures: determining the risk of blunt cardiac injury. Ann R Coll Surg Engl 2000;82:162-6.

4. de Oliveira M, Hassan TB, Sebewufu R, Finlay D, Quinton DN. Long-term morbidity in patients suffering a sternal fracture following discharge from the A and E department. Injury 1998;29:609-12.

5. Carter GT, Duong V, Ho S, Ngo KC, Greer CL, Weeks DL. Side effects of commonly prescribed analgesic medications. Phys Med Rehabil Clin N Am 2014;25:457-70.

6. Oyetunji TA, Jackson HT,Obirieze AC, Moore D, Branche MJ, Greene WR, et al. Associated injuries in traumatic sternal fractures: a review of the National Trauma Data Bank. Am Surg 2013;79:702-5.

7. Raghunathan R, Porter K. Sternal fractures. Trauma 2009;11:77-92.

8. Perez MR, Rodriguez RM, Baumann BM, Langdorf MI, Anglin D, Bradley RN, et al. Sternal fracture in the age of pan-scan. Injury 2015;46:1324-7.

9. Terry NL, Watts JR Jr, Sonavane SK, Beasley TM, Munden R, Nath PH, et al. Improved identification of sternal injuries with multidetector computed tomography (MDCT): sagittal reconstructions. Clin Imaging 2016;40:739-44.

10. Nickson C, Rippey J. Ultrasonography of sternal fractures. Australas J Ultrasound Med 2011;14:6-11. 
11. Racine S, Émond M, Audette-Côté JS, Le Sage N, Guimont C, Moore L, et al. Delayed complications and functional outcome of isolated sternal fracture after emergency department discharge: a prospective, multicentre cohort study. CJEM 2016;18:349-57.

12. Raza I, Narayanan M, Venkataraju A, Ciocarlan A. Bilateral Subpectoral Interfascial Plane Catheters for Analgesia for Sternal
Fractures: A Case Report. Reg Anesth Pain Med 2016;41:607-9.

13. Burns LT, Beasley DA, Stevens MA, Crabtree DE, Mehaffey GR. Pectointercostal Fascial Block Catheters for Thoracic Injuries: A Case Series. A A Pract 2018;11:340-3.

14. Bondár A, Szucs S, Iohom G. Thoracic paravertebral blockade. Med Ultrason 2010;12:223-7. 